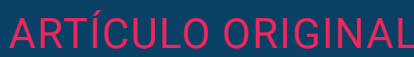

\title{
Insuficiencia renal aguda en pacientes tratados con aminoglucósidos
}

\author{
Recibido: 17/7/19 Aceptado: 12/1/20
}

Lucía Lamponi Tappatá1, Ana Laura Tomás², Joaquín Hartstock², Lisandro Prieto², Carolina Pfeiffer², María Florencia Gallardo², Ana Julia Altube², María Paula Sofio², Romina Dodero ${ }^{2}$ y Diego Maurizi

\section{RESUMEN}

Introducción: El riesgo de desarrollo de insuficiencia renal asociada al uso de aminoglucósidos es muchas veces una limitación para su uso en muchos profesionales de la salud. El objetivo de este trabajo fue conocer la frecuencia de insuficiencia renal en pacientes tratados con aminoglucósidos y los posibles factores asociados a su aparición.

Materiales y métodos: Estudio descriptivo, longitudinal y prospectivo. Se incluyeron pacientes que recibieron aminoglucósidos por al menos 72 horas. Se realizó dosaje de creatinina basal y cada 48 horas durante el tratamiento antibiótico y luego de siete días de finalizado el mismo o posterior. Se definió insuficiencia renal aguda como el aumento de creatinina de $0,3 \mathrm{mg} / \mathrm{dl}$ con respecto a la basal.

Resultados: Se analizaron datos de 107 pacientes y se halló una frecuencia de insuficiencia renal de $10,3 \%$, siendo 5,8\% durante el tratamiento aminoglucósido y 4,5\% luego de la suspensión del mismo. Los pacientes que desarrollaron insuficiencia renal tenían mayores dosis de aminoglucósidos con respecto al grupo que no presentó dicho evento, siendo esta diferencia estadísticamente significativa.

Conclusiones: La frecuencia de insuficiencia renal en pacientes tratados con aminoglucósidos resultó dentro de los intervalos descritos en la literatura y la mayoría de los pacientes presentaron recuperación completa de la

\author{
'Unidad de infectología del Hospital Municipal de Agudos \\ Dr. Leónidas Lucero, Bahía Blanca, provincia de Buenos \\ Aires, Argentina. \\ ${ }^{2}$ Servicio de clínica médica del Hospital Municipal de \\ Agudos Dr. Leónidas Lucero, Bahía Blanca, provincia de \\ Buenos Aires, Argentina. \\ Dirección para la correspondencia: Lucía Lamponi \\ Tappatá. Florida 1323, dep D, Bahía Blanca, provincia \\ de Buenos Aires, Argentina (CP 8000). \\ Email: lucialamponit@gmail.com \\ El protocolo de trabajo fue aprobado por un comité de \\ ética y de investigación y se obtuvo consentimiento \\ informado de los pacientes incluidos. \\ Los autores declaran que no existieron fuentes de \\ financiamiento ni conflictos de interés entorno a \\ esta publicación. \\ Este trabajo es original y no ha sido presentado ni \\ publicado anteriormente.
}

función renal. Consideramos que la insuficiencia renal no supone un efecto adverso clínicamente significativo si los aminoglucósidos se usan con las precauciones adecuadas. Esto, sumado a sus buenos resultados clínicos y al bajo impacto en la ecología bacteriana, los convierte hoy en día en antibióticos de uso frecuente en nuestro hospital.

Palabras clave: aminoglucósidos, lesión renal aguda, agentes antibacterianos. 


\section{Introducción}

Los aminoglucósidos son antibióticos de amplio espectro que actúan a través de la inhibición de la síntesis de proteínas (1). Desde su introducción en 1944, los aminoglucósidos fueron ampliamente utilizados, cayendo en desuso en las últimas décadas debido al aumento en la disponibilidad de cefalosporinas, carbapenémicos y fluoroquinolonas, los cuales poseen mayor cobertura antimicrobiana y menores toxicidades. Sin embargo, el aumento de la resistencia a estas clases de fármacos ha llevado a un renovado interés en los aminoglucósidos (1). Estos antibióticos poseen alta efectividad, bajo costo y no generan gran daño en la ecología bacteriana del paciente (2).

Actualmente, la principal limitación para el uso de aminoglucósidos es el riesgo de nefrotoxicidad, el cual está relacionado con la acumulación de la droga en la corteza renal, que podría generar alteración en la síntesis proteica de la membrana de las células tubulares, culminando en su apoptosis $(1,3,4)$. Se estima que esta posible complicación tiene una frecuencia del 10 al $20 \%$ cuando el daño renal se define por un aumento en la concentración de creatinina plasmática de 0,5 a $1 \mathrm{mg} / \mathrm{dl}$ o un aumento del $50 \%$ de la concentración de creatinina basal (3). Sin embargo, otros estudios que analizaron la administración de aminoglucósidos en una sola dosis diaria reportan una incidencia de insuficiencia renal menor, que va de 0 a $14 \%$ (1).

En general, la nefrotoxicidad se observa entre el quinto y séptimo día de iniciado el tratamiento antibiótico, y dado que los túbulos proximales pueden regenerarse, el curso usual es la recuperación de la función renal, generalmente dentro de los 21 días de suspendido el tratamiento. El daño renal irreversible no es común, pero el mismo podría ocurrir con tratamientos prolongados, incluso con dosis bajas de aminoglucósidos (3).

Se han descripto varios factores de riesgo para el desarrollo de nefrotoxicidad por aminoglucósidos, siendo los mayormente implicados la duración prolongada del tratamiento antibiótico, la mayor frecuencia de dosis, la concentración plasmática elevada del antibiótico, la edad avanzada, las enfermedades concomitantes (diabetes, enfermedad renal crónica, enfermedad hepática avanzada y leucemia), la disminución del volumen arterial efectivo, la sepsis y los fármacos nefrotóxicos asociados $(5,6)$.

Los aminoglucósidos son fármacos ampliamente utilizados en nuestro hospital para múltiples infecciones (abdominales, urinarias, de partes blandas, sepsis sin foco, entre otras), observándose buenos resultados clínicos, con baja percepción de complicaciones. El objetivo de este trabajo fue conocer la frecuencia de insuficiencia renal en pacientes tratados con aminoglucósidos y los posibles factores asociados a su desarrollo.

\section{Materiales y métodos}

Estudio descriptivo, longitudinal y prospectivo. La población de estudio fueron pacientes internados en los servicios de clínica médica y clínica quirúrgica del Hospital Municipal de Agudos Dr. Leónidas Lucero de la cuidad de Bahía Blanca, que recibieron tratamiento con aminoglucósidos entre junio de 2017 y febrero de 2018. Se incluyeron pacientes que firmaron consentimiento informado y que tuvieron una creatinina basal previo al inicio del tratamiento antibiótico. Se excluyeron aquellos en diálisis crónica o que luego de finalizado el seguimiento hubieran recibido aminoglucósidos por menos de 72 horas. Fueron excluidos del presente análisis los pacientes con información incompleta.

Se definió insuficiencia renal aguda como un aumento de creatinina plasmática de $0,3 \mathrm{mg} / \mathrm{dl}$ con respecto a la medición basal, según los criterios AKIN (7). Se realizó dosaje de creatinina cada 48 horas durante el tratamiento y al menos un control de creatinina siete días luego de finalizado el mismo o posterior. Se efectuó el seguimiento prospectivo de los pacientes registrando las variables de estudio clínicas y de laboratorio de acuerdo al protocolo. Si bien las normas hospitalarias de uso de aminoglucósidos sistematizan las infecciones en las cuales indicar estos antibióticos, la dosis (gentamicina 3 a $5 \mathrm{mg} / \mathrm{kg} / \mathrm{día}$ y amikacina $15 \mathrm{mg} / \mathrm{kg} / \mathrm{día}$, ambas de acuerdo al peso ideal, en monodosis, con ajuste de acuerdo a la función renal) y la duración de los tratamientos, estas variables quedaron sujetas a la decisión del médico tratante, sin intervención por parte de los investigadores.

Se diseñó una planilla para el registro de las siguientes variables de estudio: edad, sexo, factores predisponentes para insuficiencia renal por aminoglucósidos (diabetes, insuficiencia renal crónica, cirrosis, leucemia), creatinina basal, índice nitrógeno ureico en sangre/creatinina (BUN/ Cr), tensión arterial menor a $90 / 60 \mathrm{mmHg}$ al momento de iniciar el antibiótico, sepsis al inicio y a las 48 horas, drogas nefrotóxicas asociadas (3), aminoglucósido elegido, dosis de antibiótico, días de tratamiento, fallo clínico o 
microbiológico y mortalidad en internación. De acuerdo a la literatura, un índice BUN/Cr $>20$ es indicativo de disminución del volumen intravascular renal (8). La sepsis fue definida de acuerdo al consenso del American College of Chest Physicians/Society of Critical Care Medicine que establece el concepto de respuesta inflamatoria sistémica (SIRS, por sus siglas en inglés) (9).

Se analizaron los datos mediante el programa estadístico SPSS 24.0. Se calculó la frecuencia de insuficiencia renal y se comparó con el grupo que no la desarrolló en términos de las variables definidas. Las mismas se expresaron como porcentajes de frecuencia o como medias con desvío estándar. Se utilizó la prueba t para muestras independientes para calcular la diferencia de medias de las variables numéricas y la prueba Chi cuadrado para las variables cualitativas. En los casos que se constató insuficiencia renal aguda, se analizó su gravedad, su reversibilidad y sus posibles causas alternativas al aminoglucósido.

\section{Resultados}

\section{Análisis de la población que utilizó aminoglucósidos}

Durante el período de estudio, 263 pacientes recibieron aminoglucósidos. Se excluyeron 129 por haber utilizado aminoglucósidos por menos de 72 hs y se eliminaron 27 personas por falta de datos, por lo que la muestra final estuvo conformada por 107 individuos.

El $58,9 \%$ de los pacientes fueron de sexo masculino, con una media de edad de 55,5 años (DE 19,3). El $29 \%$ de los pacientes tuvo algún antecedente considerado factor de riesgo para desarrollar insuficiencia renal por aminoglucósidos, siendo la diabetes el principal.

En el $29,2 \%$ de los casos el aminoglucósido fue elegido para el tratamiento de infecciones abdominales, en el $28,3 \%$ cubriendo foco en piel, en el $26,4 \%$ para infecciones urinarias, en el $7,5 \%$ para sepsis sin foco, seguido de otros usos con menor frecuencia. En el $90,7 \%$ de los pacientes el antibiótico se inició de forma empírica. El tiempo entre el ingreso hospitalario y el inicio del aminoglucósido fue en promedio de 2,2 días (DE 6).

Al momento de iniciar el antibiótico, $54,9 \%$ de los pacientes tuvieron un índice BUN/Cr mayor a $20,15 \%$ estaban con tensión arterial menor a $90 / 60$ y el $35,3 \%$ presentaba criterios de sepsis. A las 48 horas, el 9,1\% de los pacientes persistían con criterios de sepsis.
El 97,2\% de los pacientes presentaban al menos una droga nefrotóxica asociada al aminoglucósido, siendo la media de 2,6 fármacos nefrotóxicos por paciente (DE 1,2).

En cuanto al tipo de aminoglucósido, el $86,9 \%$ de los pacientes utilizó gentamicina, 9,3\% amikacina y 3,8\% ambos antibióticos de forma secuencial. La gentamicina y la amikacina fueron administradas en todos los casos en forma de monodosis diaria, siendo la media de dosis de gentamicina de $237,5 \mathrm{mg} /$ día (DE 34,7) y de amikacina de $904,7 \mathrm{mg} /$ día (DE 183,8). El promedio de tiempo de uso de aminoglucósido fue de 5,8 días (DE 2,6).

Ocho pacientes $(7,5 \%)$ que utilizaron aminoglucósidos presentaron fracaso clínico, pudiendo constatarse en seis de ellos absceso o colección no drenada y en uno mala perfusión en el miembro afectado. En tres pacientes $(2,8 \%)$ en los que se había elegido el aminoglucósido de forma empírica se constató fracaso microbiológico, ya que se asiló un germen resistente al mismo, requiriendo modificación del esquema antibiótico.

El 13,1\% de los pacientes que utilizaron aminoglucósidos tenían insuficiencia renal basal al momento del inicio del antibiótico, definida como creatinina mayor a $1,2 \mathrm{mg} / \mathrm{dl}$. De estos pacientes, ninguno presentó empeoramiento de la función renal luego de iniciado el aminoglucósido.

\section{Análisis de los pacientes que desarrollaron insuficiencia renal aguda}

Un 10,3\% de los pacientes que recibieron aminoglucósidos presentaron insuficiencia renal como complicación, $5,8 \%$ durante el uso del antibiótico y $4,5 \%$ posterior a la suspensión del mismo. De estos pacientes ninguno tenía insuficiencia renal al momento de iniciar el aminoglucósido.

De los seis pacientes que presentaron insuficiencia renal durante el tratamiento, tres lo hicieron en contexto de fallo multiorgánico y muerte. En los tres pacientes restantes el aumento de creatinina fue de 0,32, 1,25 y 0,32 mg/dl con respecto a la basal y la recuperación fue completa a las 24 horas, 48 horas y 30 días respectivamente. La falla renal de la paciente que presentó el mayor aumento de creatinina fue en contexto de shock séptico (hipotensión arterial, anuria y acidosis metabólica) por colección intraabdominal no resuelta. El aumento de creatinina de estos pacientes se produjo en promedio a los 5,5 días (DE $3,7)$ de iniciado el tratamiento antibiótico. 
En cuanto a los pacientes que presentaron insuficiencia renal luego de finalizado el tratamiento con aminoglucósido, la misma fue en promedio a los 10,4 días (DE 3,7) de suspendido el antibiótico. En estos cinco pacientes, el promedio de aumento fue de $0,41 \mathrm{mg} / \mathrm{dl}(\mathrm{DE} 0,11)$ con respecto a la creatinina basal, y la recuperación fue completa en cuatro de ellos, desconociéndose la evolución del paciente restante (ver Tabla 1). La recuperación de la función renal fue en promedio a los 19 días (DE 27,4).

\section{Comparación de subgrupos}

En la comparación entre el grupo de pacientes que presentó insuficiencia renal intra o post tratamiento $(n=11)$ y el grupo

\section{Tabla 1. Pacientes que desarrollaron insuficiencia renal aguda (IRA) durante el tratamiento o} luego de la suspensión del mismo

\begin{tabular}{|c|c|c|c|c|c|c|c|c|c|}
\hline $\mathbf{N}^{\circ}$ & Cr basal & IRA basal & $\begin{array}{l}\text { Mayor } \mathrm{Cr} \\
\text { intra - tto }\end{array}$ & $\begin{array}{l}\text { Aumento de } \mathrm{Cr} \text { con } \\
\text { respecto a basal }\end{array}$ & $\begin{array}{c}\text { IRA } \\
\text { intra - tto }\end{array}$ & $\begin{array}{c}\mathrm{Cr} \\
\text { post tto }\end{array}$ & $\begin{array}{l}\text { Aumento de } \mathrm{Cr} \text { con } \\
\text { respecto a basal }\end{array}$ & $\begin{array}{c}\text { IRA } \\
\text { post tto }\end{array}$ & $\begin{array}{l}\text { Cr en la } \\
\text { evolución }\end{array}$ \\
\hline 1 & 1,09 & No & 1,65 & 0,56 & Sí & Falleció & - & No & - \\
\hline 2 & 0,66 & No & 1,91 & 1,25 & Sí & 0,54 & - & No & - \\
\hline 3 & 0,62 & No & 1,09 & 0,47 & Sí & 0,80 & - & No & - \\
\hline 4 & 0,78 & No & 1,25 & 0,47 & Sí & Falleció & - & No & - \\
\hline 5 & 0,56 & No & 0,90 & 0,34 & Sí & Falleció & - & No & - \\
\hline 6 & 0,65 & No & 0,97 & 0,32 & Sí & 0,86 & - & No & - \\
\hline 7 & 0,89 & No & 1,03 & - & No & 1,20 & 0,31 & $\mathrm{Si}$ & 1,08 \\
\hline 8 & 0,39 & No & 0,39 & - & No & 0,72 & 0,33 & Si & 0,25 \\
\hline 9 & 0,55 & No & 0,55 & - & No & 0,89 & 0,34 & $\mathrm{Si}$ & - \\
\hline 10 & 0,75 & No & 0,65 & - & No & 1,29 & 0,54 & Si & 0,85 \\
\hline 11 & 0,72 & No & 0,64 & - & No & 1,25 & 0,53 & $\mathrm{Si}$ & 0,65 \\
\hline
\end{tabular}

Cr: creatinina $(\mathrm{mg} / \mathrm{dl})$

Tto: tratamiento

\section{Tabla 2. Comparación de subgrupos: pacientes que presentaron insuficiencia renal aguda (IRA)} vs pacientes que no la presentaron (no IRA)

\begin{tabular}{|l|c|c|c|} 
& Pacientes IRA (n=11) & Pacientes no IRA (n=96) & P \\
\hline Edad & 62,8 & 54,6 & 0,09 \\
\hline Sexo femenino & $8(72,7 \%)$ & $36(37,5 \%)$ & $\mathbf{0 , 0 2}$ \\
\hline DBT & $4(36,4 \%)$ & $25(26 \%)$ & 0,47 \\
\hline Índice BUN/Cr & 24,9 & 21,9 & 0,17 \\
\hline Índice BUN/Cr > 0 & $7(63,6 \%)$ & $43(44,8 \%)$ & 0,34 \\
\hline Hipotensión & 0 & $15(15,6 \%)$ & - \\
\hline Sepsis al inicio & $3(27,3 \%)$ & $34(35,4 \%)$ & 0,6 \\
\hline Sepsis a las 48 hs & 0 & $7(7,3 \%)$ & - \\
\hline Media drogas nefrotóxicas asociadas & 2,4 & 2,6 & 0,25 \\
\hline Uso de gentamicina & $9(81,8 \%)$ & $84(87,5 \%)$ & 0,6 \\
\hline Dosis media gentamicina (mg/día) & 262,2 & 234,9 & $\mathbf{0 , 0 1}$ \\
\hline Uso de amikacina & $2(18,2 \%)$ & $8(8,3 \%)$ & 0,29 \\
\hline Dosis media amikacina (mg/día) & 821,5 & 937,5 & 0,23 \\
\hline Ambos ATB de forma secuencial & 0 & 4 & - \\
\hline Días de tratamiento & 6,5 & 5,7 & 0,15 \\
\hline Insuficiencia renal basal & 0 & $14(14,6 \%)$ & - \\
\hline Fallecimiento & $3(27,3 \%)$ & $5(5,2 \%)$ & $\mathbf{0 , 0 0 8}$
\end{tabular}


que no presentó este evento $(n=96)$ se objetivó que el primer grupo fue con mayor frecuencia del sexo femenino ( $72,7 \%$ vs $37,5 \%)$, utilizó dosis superiores de gentamicina (media 262,2 vs $234,8 \mathrm{mg} /$ día) y tuvo mayor mortalidad ( 27,3 vs $5,2 \%)$, todas con diferencia estadísticamente significativa. No se encontraron diferencias entre los subgrupos en cuanto a las demás variables (ver Tabla 2).

\section{Discusión}

La frecuencia de insuficiencia renal en pacientes tratados con aminoglucósidos hallada en este trabajo estuvo dentro del rango reportado en la bibliografía $(1,3)$. Si bien este estudio mostró un $10,3 \%$ de insuficiencia renal (11 pacientes), se pudo concluir que al menos cuatro de los pacientes tuvieron otra causa de fallo renal agudo, disminuyendo así la frecuencia de insuficiencia renal asociada a aminoglucósidos a 6,5\%. Por otro lado, si tomamos en cuenta únicamente la insuficiencia renal de aparición durante el tratamiento aminoglucósido, como analizaron la mayor parte de los trabajos publicados, observamos que la misma desciende a 5,9\%. Por último, es importante considerar que en la mayoría de los estudios de la literatura se usaron puntos de corte de creatinina más elevados para definir insuficiencia renal aguda (3). Utilizando un aumento de $0,5 \mathrm{mg} / \mathrm{dl}$ de creatinina, la frecuencia de insuficiencia renal en este trabajo hubiese sido solo del $3,9 \%$.

En este estudio los pacientes presentaron insuficiencia renal en promedio a los 5,5 días del inicio del antibiótico, lo cual coincide con los cinco a siete días reportados en la literatura (3). Además, tal como muestra la bibliografía, en la mayoría de los casos se pudo constatar que la falla renal fue transitoria y con recuperación completa posterior (siete de los pacientes recuperaron completamente, tres fallecieron y en uno no se pudo determinar evolución). La recuperación de la función renal en estos pacientes se constató en promedio a los 19 días de producido el fallo, valor que se encuentra dentro de los 21 días que han sido descritos en la literatura (3).

Otro hallazgo de esta investigación coincidente con lo descrito en la bibliografía fue la asociación entre mayores dosis de antibiótico aminoglucósido y desarrollo de insuficiencia renal $(5,6)$. Aunque en nuestra institución no contamos con la posibilidad de dosar aminoglucósidos, podríamos inferir que los pacientes con dosis mayores de antibióticos probablemente tuvieron mayores concentraciones séricas.

Los pacientes del grupo que desarrolló insuficiencia renal aguda tuvieron mayor mortalidad que los que no tuvieron dicho evento. Esto probablemente pueda ser explicado porque los pacientes que fallecieron en el primer grupo desarrollaron insuficiencia renal aguda en el contexto de fallo orgánico y no a causa del aminoglucósido.

No se encontraron otros factores asociados al desarrollo de insuficiencia renal, posiblemente debido a que la muestra fue pequeña para mostrar diferencias significativas. En cuanto al intervalo entre dosis de aminoglucósidos, se sabe que la administración en una única dosis diaria tiene menor probabilidad de causar nefrotoxicidad que la administración en dos dosis diarias (10). En nuestro trabajo, todos los pacientes que utilizaron aminoglucósidos lo hicieron en monodosis diaria.

Los aminoglucósidos continúan siendo antibióticos de gran utilidad en nuestro medio ya que se pudo observar que prácticamente no existió falla clínica (cuando la hubo fue en contexto de no remoción de un foco infeccioso) y se objetivó menos de 3\% de fallo microbiológico, constatando así un buen perfil de sensibilidad local.

Una de las limitaciones de este trabajo fue la pérdida de pacientes debido a la falta de datos para su análisis, principalmente por no haber aceptado participar en el estudio y no tener los dosajes de creatininas necesarias para el análisis del comportamiento de la función renal.

\section{Conclusiones}

El amplio espectro de actividad, su rápida acción bactericida, las propiedades farmacocinéticas favorables y el menor potencial de daño ecológico convierten a los aminoglucósidos en una clase de antibióticos clínicamente útiles en numerosos tipos de infecciones. Este trabajo demuestra que la insuficiencia renal en pacientes que usan aminoglucósidos es un evento relativamente frecuente en la práctica diaria pero con poca relevancia clínica en la evaluación costo-beneficio. Consideramos que los aminoglucósidos son drogas seguras si se realiza un uso individualizado, ajustando las dosis de acuerdo a la función renal y la presencia de factores de riesgo para nefrotoxicidad, minimizando las drogas nefrotóxicas concomitantes, asegurando un adecuado volumen intravascular, utilizando tratamientos cortos y realizando dosaje de las concentraciones séricas en caso de ser posible. 


\section{Referencias}

1. Kevin M. Krause, Alisa W. Serio, Timothy R. Kane, Lynn E. Connolly. Aminoglycosides: An Overview. Cold Spring Harb Perspect Med. 2016 Jun; 6(6): a027029.

2. Kumana,C. R., Yuen, K.Y.(1994). Parenteral aminoglycoside theraphy. Selection, administration and monitoring. Drugs. 47 (6): 902-913.

3. Molitoris BA. Manifestations of and risk factors for aminoglycoside nephrotoxicity. Last uptodate, jun 2017.

4. Lameier N., Ekanoian G. (2012). Clinical Practice Guideline for the evaluation and management of chronic kidney disease. Official Journal of the International Society of Nephrology. 2: 61 - 63.

5. Humes, H. D. (1988). Aminoglycoside nephrotoxicity. Kidney Int. 33 (4) 900- 911.

6. Moore R.D, Smith C.R., Lipsky J.J, Mellits E.D, Lietman P.S. (1984). Risk factors for nephrotoxicity in patients treated with aminoglycosides. Ann Intern Med. 100 (3): 352- 357.

7. Mehta RL, Kellum JA, Shah SV, Molitoris BA, Ronco C, Warnock DG, et al. Acute Kidney Injury Network: report of an initiative to improve outcomes in acute kidney injury. Crit Care. 2007;11(2):R31

8. Restrepo Valencia CA (editor), Buitrago Villa CA, Torres Saltarín JJ, Serna Flórez J. Libro Nefrología básica 2. Capítulo 2. Asociación Colombiana de Nefrología e Hipertensión Arterial. Manizales [Colombia] : La Patria. Segunda edición, año 2012.

9. American College of Chest Physicians/Society of Critical Care Medicine Consensus Conference: definitions for sepsis and organ failure and guidelines for the use of innovative therapies in sepsis. Crit Care Med. 1992 Jun;20(6):864-74.

10. Rybak M.J, Abate B.J, Kang S.L, Ruffing M.J, Lerner S.S, Drusano G.L. (1999). Prospective evaluation of the effect of an aminoglycoside dosing regimen on rates of observed nephrotoxicity and ototoxicity. Antimicrob Agents Chemother. 43: 1549 - 1555. 
Acute kidney injury in patients treated with aminoglycosides

The risk of developing renal failure associated with the use of aminoglycosides is often a limitation for its use in many health professionals. The objective of this study was to know the frequency of renal failure in patients treated with aminoglycosides and the possible factors associated with their appearance.

Materials and methods: descriptive, longitudinal and prospective study. Patients who received aminoglycosides for at least 72 hours were included. Basal creatinine was measured and every 48 hours during the antibiotic treatment and after 7 days or more of its suspension. Acute renal failure was defined as an increase in creatinine of $0.3 \mathrm{mg} / \mathrm{dl}$ compared to baseline. Results: data from 107 patients were analyzed, and the frequency of renal failure was $10.3 \%$, being $5.8 \%$ during the treatment with aminoglycoside and $4.5 \%$ after its suspension. The patients who developed renal failure had higher doses of aminoglycoside respect to the group that did not present this event, being this difference statistically significant. Conclusions: the frequency of renal failure in patients treated with aminoglycosides was within the ranges described in the literature and the majority of patients had complete recovery of renal function. We believe that renal failure does not represent a clinically significant adverse effect if aminoglycosides are used with adequate precautions. This, added to their good clinical results and low impact on bacterial ecology, makes them very used antibiotics today in our hospital.

Key words: aminoglycosides, acute kidney injury, anti-bacterial agents. 